\title{
Relación de oralidad y las decisiones judiciales en los procesos de alimentos tramitados en el segundo juzgado de paz letrado de Tarapoto, 2019
}

\section{Relationship of orality and judicial decisions in the processes of food processed in the second court of peace letter of Tarapoto, 2019}

Deza Padilla, Juan Fernando 10000-0003-0110-2052]

${ }^{1}$ Universidad Nacional de San Martín, Tarapoto, Perú juanfernandodeza@gmail.com

Resumen. El presente trabajo denominado relación de oralidad y las decisiones judiciales en los procesos de alimentos tramitados en el Segundo Juzgado de Paz Letrado de Tarapoto, 2019, tiene como objetivo determinar la relación existente entre oralidad y las decisiones judiciales en los procesos de alimentos tramitados en el Segundo Juzgado de Paz Letrado de Tarapoto, 2019. El tipo de investigación es pura, su nivel de investigación es descriptiva correlacional, la muestra tomada fue conformada por 40 expedientes judiciales físicos, con decisión judicial de primera instancia, emitidos entre los meses de marzo abril y mayo del año 2019. Determinándose el coeficiente de correlación de Pearson de 0,862793, concluyéndose así que existe una correlación positiva alta, entre ambas variables de estudio. Asimismo, se determinó la relación existente entre cada variable y sus dimensiones, concluyéndose que en la relación más significativa entre variable oralidad y sus dimensiones fue la dimensión alegatos, la cual presentó una relación mucho más significativa en la oralidad desarrolladas en audiencia única de alimentos.

Palabras clave: Alegatos, decisiones judiciales, proceso de alimentos, obligación alimentaria, oralidad

Citar como: Deza Padilla, J. F. (2021). Relación de oralidad y las decisiones judiciales en los procesos de alimentos tramitados en el segundo juzgado de paz letrado de Tarapoto, 2019. Revista Científica Ratio Iure, 1(2), 38-48. https://doi.org/10.51252/rc ri.v1i2.196

Recibido: 18/04/2021

Aceptado: $19 / 06 / 2021$

Publicado: 28/07/2021
Abstract. The present work called the orality relationship and the judicial decisions in the food processes processed in the Second Justice Court of Tarapoto, 2019, aims to determine the relationship between orality and judicial decisions in the food processes processed in the Second Justice Court of Tarapoto, 2019; The type of investigation is pure, its level of investigation is descriptive correlational, the sample taken was made up of 40 physical judicial files, with judicial decision of first instance, issued between the months of March April and May of the year 2019. Determining the coefficient of Pearson's correlation of 0.862793 , concluding that there is a high positive correlation between the two study variables. Likewise, the relationship between each variable and its dimensions was determined, concluding that in the most significant relationship between variable orality and its dimensions was the allegations dimension, which presented a much more significant relationship in orality developed in a single food audience. Keywords: Allegations, food process, food obligation, judicial decisions, orality 


\section{$1 \quad$ Introducción}

Los cambios que se gestan en la actualidad están ligados al uso de la tecnología de la información, y el derecho adopta dentro de su desarrollo dichos cambios que le permiten hacer más simple y preciso la labor de los operadores de justicia, abarcando hasta llegar al proceso de alimentos, materia, con más números de casos que llegan a ventilarse en los juzgados de Paz Letrados.

A nivel internacional como nacional los derechos de los niños y adolescentes han sido de gran preocupación y de suma importancia, por ende, las Naciones Unidas, las organizaciones internacionales, han promovido diversos instrumentos internacionales destinados a regular materias como la competencia judicial, el reconocimiento y las decisiones judiciales, en la mejora para la protección de esta rama del Derecho.

La Defensoría del Pueblo, ha realizado un informe en el año 2018 en lo que concierne a la conclusión del proceso, se determinó que, de los 3,512 casos analizados sobre alimentos, se obtuvo que el $67,9 \%$ de los expedientes culminaron con sentencia, y solo 4,7\% concluyó mediante conciliación y lo demás con abandono y otros. Defensoría de Pueblo (2018).

Frente a ello el Segundo Juzgado de Paz Letrado viene desarrollando audiencias grabadas en audio y video, para que las partes de alguna manera encuentren una mejor alternativa para la absolución de sus procesos judiciales mediante la oralidad.

Si una madre o padre, como representante del menor alimentista acude al órgano judicial e interponer una demanda, no está interviniendo precisamente en el proceso como la parte fuerte de la relación procesal; puesto que el demandado ha defraudado con su incumplimiento de obligación de padre.

Para ello es necesario simplificar y flexibilizar el formalismo en procesos de alimentos, con el apoyo de la oralidad que recorta, objetiviza la etapa procesal, concentra las actuaciones que se generan dentro del proceso en la etapa de audiencia y mejora la gestión judicial, la salida al problema es realizar un encuentro de los sujetos procesales mediante una audiencia en la que todas los actos procesales se oralicen dentro de la etapa de trámite, sería una innovación dentro del proceso de alimentos si contamos además de equipos de audio y video que permitan grabar y perennizar las audiencias además permitan dar rapidez a la resolución del conflicto a través de las decisiones judiciales que pone fin al proceso.

En la oralidad se utilizan principios procesales como la inmediación, concentración, con los cuales se enfocarían los pedidos de manera precisa y objetiva, respecto del aporte que puedan proponer los sujetos procesales perdimiento que las decisiones judiciales serían adecuadas. 
En la presente investigación se determinó la relación entre oralidad y las decisiones judiciales producto de las audiencias únicas dentro del proceso de alimentos que fueron tramitados en el Segundo Juzgado de Paz Letrado de Tarapoto, en los meses de marzo, abril y mayo del año 2019.

\subsection{Formulación del Problema.}

¿Cuál es la relación entre la oralidad y las decisiones judiciales en los procesos de alimentos tramitados en el Segundo Juzgado de Paz Letrado de Tarapoto, 2019?

\subsection{Objetivo general:}

El objetivo general, como tres específicos, teniéndose como objetivo general: Determinar cuál es relación entre oralidad y las decisiones judiciales en los procesos de alimentos tramitados en el Segundo Juzgado de Paz Letrado de Tarapoto, 2019.

\subsection{Hipótesis.}

Existe relación positiva muy alta entre la oralidad y las decisiones judiciales en los procesos de alimentos tramitados en el Segundo Juzgado de Paz Letrado de Tarapoto, 2019

\subsection{Objetivos específicos.}

- Conocer como es la oralidad en los procesos de alimentos en el Segundo Juzgado de Paz Letrado, 2019.

- Identificar las decisiones judiciales en los procesos de alimentos en el Segundo Juzgado de Paz Letrado, año 2019.

- Determinar la relación entre la oralidad y las decisiones judiciales en los procesos de alimentos tramitados en el Segundo Juzgado de Paz Letrado de Tarapoto, 2019, en todas sus dimensiones.

\section{Materiales y Métodos}

Cabe señalar también que, para la presente investigación, se tomó en cuenta los expedientes judiciales físicos que fueron tramitados en el Segundo Juzgado de Paz Letrado de Tarapoto en los meses comprendidos desde enero a junio y las audiencias grabadas en un dispositivo de audio que permitió conocer la variable de la oralidad.

La muestra estuvo confirmada por 40 expedientes que fueron seleccionados utilizando el muestreo subjetivo por decisión razonada por las características que poseen los expedientes de la muestra, a los cuales se les aplico la guía de análisis de datos, conformado por 17 ítems para la variable oralidad y de 8 ítems para la variable decisiones judiciales. 


\subsection{Análisis de datos.}

Para una comprobación de la hipótesis, los datos recolectados fueron procesados y analizado a través del Microsoft Excel y el programa estadístico IBM SPSS STATISTICS 25, gracias al coeficiente de correlación de Pearson. La misma que se graficó de la siguiente formula:

$$
r=\frac{N \sum \mathbf{X Y}-\left(\sum \mathbf{X}\right)\left(\sum \mathbf{Y}\right)}{\sqrt{\left[N \sum \boldsymbol{y}^{2}-\left(\sum \mathbf{Y}\right)^{2}\right]\left[N \sum \boldsymbol{x}^{2}-\left(\sum \mathbf{X}\right)^{2}\right]}}
$$

\section{Donde:}

$\mathrm{N} \quad$ : Es el total de la muestra.

$\sum \mathrm{XY}$ : Es la sumatoria del producto de las variables uno y dos

¿X : Representa la sumatoria de la Variable Uno, oralidad

$\sum \mathrm{Y} \quad$ Representa la sumatoria de la variable dos, decisiones judiciales.

El coeficiente de correlación de Pearson puede tomar valores entre +1 y -1 . El primero se da cuando existe correlación positiva perfecta, el segundo, cuando hay correlación negativa perfecta. Briones, (2012) 


\section{Resultados y discusiones}

Tabla 1

Relación entre la oralidad y las decisiones judiciales en los procesos de alimentos tramitados en el Segundo Juzgado de Paz Letrado de Tarapoto, 2019

\begin{tabular}{|c|c|c|c|}
\hline $\mathrm{N}^{\circ}$ & EXP. & V1 & $\mathrm{V} 2$ \\
\hline 1 & 160-2019-FC & 43 & 16 \\
\hline 2 & 165-2019-FC & 37 & 18 \\
\hline 3 & 194-2019-FC & 42 & 20 \\
\hline 4 & 223-2019-FC & 31 & 12 \\
\hline 5 & 227-2019-FC & 39 & 12 \\
\hline 6 & 241-2019-FC & 37 & 17 \\
\hline 7 & 235-2019-FC & 34 & 16 \\
\hline 8 & 310-2019-FC & 30 & 11 \\
\hline 9 & 266-2019-FC & 30 & 12 \\
\hline 10 & 285-2019-FC & 35 & 14 \\
\hline 11 & 270-2019-FC & 25 & 11 \\
\hline 12 & 298-2019-FC & 39 & 13 \\
\hline 13 & 319-2019-FC & 24 & 11 \\
\hline 14 & 342-2019-FC & 17 & 8 \\
\hline 15 & 340-2019-FC & 41 & 19 \\
\hline 16 & 352-2019-FC & 34 & 14 \\
\hline 17 & 276-2019-FC & 37 & 15 \\
\hline 18 & 125-2019-FC & 33 & 15 \\
\hline 19 & 233-2019-FC & 35 & 11 \\
\hline 20 & 304-2019-FC & 36 & 14 \\
\hline 21 & 302-2019-FC & 41 & 16 \\
\hline 22 & 225-2019-FC & 40 & 18 \\
\hline 23 & 725-2018-FC & 35 & 13 \\
\hline 24 & 217-2019-FC & 37 & 18 \\
\hline 25 & 321-2019-FC & 49 & 20 \\
\hline 26 & 280-2019-FC & 45 & 21 \\
\hline 27 & 253-2019-FC & 46 & 24 \\
\hline 28 & 306-2019-FC & 25 & 11 \\
\hline 29 & 139-2019-FC & 19 & 10 \\
\hline 30 & 408-2019-FC & 49 & 21 \\
\hline 31 & 386-2019-FC & 27 & 13 \\
\hline 32 & 380-2019-FC & 27 & 15 \\
\hline 33 & 370-2019-FC & 36 & 15 \\
\hline 34 & 393-2019-FC & 48 & 21 \\
\hline 35 & 079-2019-FC & 45 & 21 \\
\hline 36 & 104-2019-FC & 22 & 11 \\
\hline 37 & 112-2019-FC & 33 & 16 \\
\hline 38 & 121-2019-FC & 50 & 21 \\
\hline 39 & 022-2019-FC & 32 & 14 \\
\hline 40 & 081-2019-FC & 41 & 21 \\
\hline
\end{tabular}


El cuadro antes señalado es resultado de los valores obtenidos del instrumento utilizado, sirvió para determinar el nivel de relación entre las variables materia de estudio, a través de la siguiente fórmula (ver Análisis de datos) teniéndose como resultado lo siguiente:

Tabla 2

Correlación entre oralidad y decisiones judiciales

\begin{tabular}{ll|l|l}
\hline & & Oralidad & $\begin{array}{l}\text { Decisiones } \\
\text { judiciales }\end{array}$ \\
\hline Oralidad & $\begin{array}{l}\text { Correlación de } \\
\text { Pearson }\end{array}$ & 1 & 0,862793 \\
\cline { 2 - 4 } & $\mathrm{N}$ & 40 & 40 \\
\hline $\begin{array}{l}\text { Decisiones } \\
\text { judiciales }\end{array}$ & $\begin{array}{l}\text { Correlación de } \\
\text { Pearson }\end{array}$ & 0,862793 & 1 \\
\cline { 2 - 4 } & $\mathrm{N}$ & 40 & 40 \\
\cline { 2 - 4 } & & & \\
\hline
\end{tabular}

De la tabla se puede determinar que existe una correlación positiva alta, puesto que el valor del coeficiente de correlación es de 0,862793 , lo que al aproximarse al 1 es una correlación positiva alta.

\section{Tabla 3}

La oralidad en los procesos de alimentos

\begin{tabular}{|c|c|c|c|c|c|}
\hline \multirow{2}{*}{$\begin{array}{c}\text { Escalas de } \\
\text { medición } \\
\text { variable uno }\end{array}$} & \multicolumn{2}{|c|}{$\begin{array}{l}\text { Intervalo de } \\
\text { clase }\end{array}$} & \multirow{2}{*}{$\mathbf{F i}$} & \multirow{2}{*}{ Hi } & \multirow{2}{*}{$\%$} \\
\hline & $\begin{array}{l}\text { Límite } \\
\text { inferior }\end{array}$ & $\begin{array}{l}\text { Limite } \\
\text { súper }\end{array}$ & & & \\
\hline Inadecuado & 16 & 27 & 8 & 0.2 & $20 \%$ \\
\hline Regular & 28 & 39 & 19 & 0.475 & $48 \%$ \\
\hline Adecuado & 40 & 51 & 13 & 0.325 & $33 \%$ \\
\hline \multicolumn{3}{|c|}{ Total } & 40 & 1 & $100 \%$ \\
\hline
\end{tabular}

En la tabla 3, se muestra los resultados del primer objetivo específico, consistente en la oralidad en los procesos de alimentos en el Segundo Juzgado de Paz Letrado, 2019. Los 40 expedientes judiciales que fueron procesados, de los cuales se encontraron 8 expedientes judiciales que, representan un $20 \%$ del total, los cuales están dentro de la escala de medición inadecuado. Existe 19 expedientes que se encuentran dentro de la escala de medición regular y representan el $48 \%$ del total de expedientes estudiados, de igual modo existen 13 expedientes que están dentro de la escala de adecuado, representado al $33 \%$ de los 40 expedientes. 


\section{Tabla 4}

Decisiones Judiciales en Materia de Alimentos.

\begin{tabular}{|c|c|c|c|c|c|}
\hline \multirow{2}{*}{$\begin{array}{l}\text { Escalas de } \\
\text { medición } \\
\text { variable dos }\end{array}$} & \multicolumn{2}{|c|}{$\begin{array}{l}\text { Intervalo de } \\
\text { clase }\end{array}$} & \multirow{2}{*}{ fi } & \multirow{2}{*}{ hi } & \multirow{2}{*}{$\%$} \\
\hline & $\begin{array}{l}\text { Límite } \\
\text { inferior }\end{array}$ & $\begin{array}{l}\text { Limite } \\
\text { súper }\end{array}$ & & & \\
\hline Inadecuado & 7 & 13 & 14 & 0.35 & $35 \%$ \\
\hline Regular & 14 & 20 & 20 & 0.5 & $50 \%$ \\
\hline Adecuado & 21 & 27 & 6 & 0.15 & $15 \%$ \\
\hline & Total & & 40 & 1 & $100 \%$ \\
\hline
\end{tabular}

En la tabla 4, se muestra los resultados del segundo objetico específico, consistente en las decisiones judiciales en los procesos de alimentos en el Segundo Juzgado de Paz Letrado, 2019. Observándose que de los 40 expedientes judiciales que fueron procesados, se encontraron que 14 expedientes judiciales representan un $20 \%$ del total, los mismos que se fueron evaluados dentro de la escala de medición como inadecuados. De otro lado existieron 20 expedientes que se encentraron dentro de la escala de medición regular y representan el $48 \%$ del total de expedientes estudiados. Por último, en cuanto a la escala de medición adecuado este fue de un $15 \%$ del total, conformado por 6 expedientes.

\section{Resumen de los resultados respecto al tercer objetivo específico.}

\section{Tabla 5}

Cuadro resumen de los resultados respecto al tercer objetivo específico

\begin{tabular}{|c|c|c|c|c|}
\hline Variables & Dimensiones & $\begin{array}{l}\text { Coeficiente de } \\
\text { determinación }\end{array}$ & $\begin{array}{l}\text { Coeficiente de } \\
\text { correlación }\end{array}$ & $\begin{array}{l}\text { Significado } \\
\text { (ver apéndice) }\end{array}$ \\
\hline \multirow{5}{*}{ 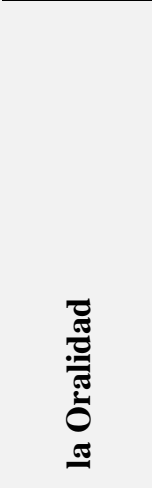 } & Inmediación & 0.6933 & 0.83264638 & $\begin{array}{l}\text { correlación } \\
\text { positiva alta }\end{array}$ \\
\hline & $\begin{array}{l}\text { Fijación de } \\
\text { puntos } \\
\text { controvertidos }\end{array}$ & 0.5127 & 0.71603073 & $\begin{array}{l}\text { correlación } \\
\text { positiva alta }\end{array}$ \\
\hline & Alegatos & 0.8272 & 0.90950536 & $\begin{array}{l}\text { correlación } \\
\text { positiva muy } \\
\text { alta }\end{array}$ \\
\hline & Publicidad & 0.6567 & 0.81037029 & $\begin{array}{l}\text { correlación } \\
\text { positiva alta }\end{array}$ \\
\hline & $\begin{array}{l}\text { Economía } \\
\text { procesal }\end{array}$ & 0.568 & 0.75365775 & $\begin{array}{l}\text { correlación } \\
\text { positiva alta }\end{array}$ \\
\hline \multirow{3}{*}{ 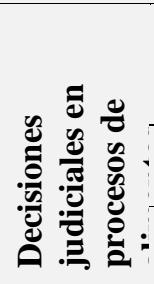 } & Conciliación & 0.245 & 0.49497475 & $\begin{array}{l}\text { correlación } \\
\text { positiva } \\
\text { moderada }\end{array}$ \\
\hline & $\begin{array}{l}\text { Sentencia } \\
\text { fundada }\end{array}$ & 0.537 & 0.73280284 & $\begin{array}{l}\text { correlación } \\
\text { positiva alta }\end{array}$ \\
\hline & $\begin{array}{l}\text { Consentida } \\
\text { la sentencia }\end{array}$ & 0.7306 & 0.85475143 & $\begin{array}{l}\text { correlación } \\
\text { positiva alta }\end{array}$ \\
\hline
\end{tabular}


En este cuadro se compararon las dimensiones de las variables juntamente con sus dimensiones, de las cuales el resultado más significativo que se encontró fue de la dimensión alegatos que pertenece a la variable Oralidad, con un coeficiente de correlación de pesaron de 0,909505, lo que significa que existe correlación positiva muy alta (ver apéndice).

\subsection{Discusión}

Los resultados de la investigación, se muestran tal como fueron encontradas en el momento de la recolección de los datos, lo obtenido responde a los comportamientos de las variables, se determinó la existencia o no de las relaciones entre variables, pudiendo ser dicha relación negativa, positiva o nula, cabe señalar que al no existir relación va a ser nula; existiendo para la presente investigación una relación positiva entre variables, además se explica cuál es el nivel de relación positiva existente entre las dos variables, ello guarda relación con el objetivo general, que permite comprobar además nuestra hipótesis planteada.

Para la contratación de hipótesis en cuanto al objetivo general, se aplicó el coeficiente de correlación de Pearson trabajándose con cada variable y sus respectivas dimensiones, todas ellas al final se expresaron en un cuadro comparativo que permitió identificar la relación entre las dimensiones de cada variable, determinándose una correlación alta y positiva.

\section{Conclusiones}

La relación existente entre la oralidad y las decisiones judiciales tramitadas en el segundo juzgado de paz letrado de Tarapoto, sobre los procesos de alimentos, es positiva alta, comprobándose así la hipótesis planteada, lográndose además el objetivo general planteado para ello se presentó un coeficiente de correlación de Pearson de 0,862793, y encuentra dentro del rango de 0,7 a 0,89 así mismo se obtuvo para el mismo objetivo general, el coeficiente de determinación de 0,7419 , que en expresión porcentual vendría a ser 74,19\%. Lo cual significa que el 74,19\% de las decisiones judiciales, se ven influencias por la variable oralidad en los procesos de alimentos en el primer semestre de 2019.

Que la oralidad de los procesos de alimentos tramitados en el Juzgado de Paz Letrado se ha encontrado que el $20 \%$ de los expedientes judiciales en materia de alimentos la oralidad fue desarrollada de manera inadecuada; que el $48 \%$ de los expedientes judiciales el desarrollo de la oralidad fue regular, y que el $30 \%$ de los expedientes judiciales estuvieron desarrollada la oralidad de manera adecuada.

Las decisiones judiciales en los procesos de alimentos se encontraron que la relación de la oralidad y sus dimensiones: Inmediación, Fijación de puntos controvertidos, alegatos, publicidad y 
economía procesal, la dimensión con más coeficiente de correlación fue la de alegatos, siendo su coeficiente de 0.90950536, correlación positiva muy alta. Mientras que en las decisiones judiciales la dimensión sentencia consentida tuvo mayor coeficiente de correlación obtuvo siendo 0,85475143 lo que significa que existe correlación positiva alta.

\section{Referencias bibliográficas}

Alcalà, Z. N. (2003). Estudios de teoría de historia del proceso. [Mexico: Univeristaria S.A.].

Osorio Omaria, A. \& Sierra Alba, L. (2015). Ventajas y desventajas de la oralidad en la justicia colombiana. caso Manizales [Colombia: Universidad Manizales].

Alvarado Velloso, A. (2019). Academia Virtual derecho.

Briones, G. (2012). Metodologìa de la investigaciòn cuantitativa, aplicada a las ciencias sociales. [Chile: ASCUN].

Carnelutti, F. (1971). Derecho y Proceso. Ediciones Juridicas Europa - America.

Carrasco, B. J. (2011). Ejecucion Provicional de las resoluciones Judiciales. [Chile].

Chiovenda, G. (2003). Curso de Derecho procesal civil. [Mexico: Oxford University Press. S.A.]

Chirinos Soto, E. \& Chirinoa Soto, F. (2012). La Constituciòn Lectura y comentarios. [Lima : Rodhas SAC].

Contreras, E. H. (2017). Flexibilización de audiencia y la protección del Interés Superior del Niño en los procesos de alimentos del $2^{\circ}$ Juzgado de Paz Letrado de Carabayllo en los periodos 2015 - 2016. [Lima, Perú].

Couture, E. J. (1958). Fundamentos del derecho Procesal Civil. [Buenos Aires: Roque Depalma].

Couture, E. J. (1979). Estudios de Derecho Procesal Civil - Tomo I. [Buenos Aires: Depalma].

Defensoria de Pueblo. (2018). El proceso de alimentos en el Perú: avances, dificultades y retos. [Lima, Perú].

Flores Soza \& Palma Collado. (2014). Una Aproximación al Principio de Oralidad en el Proceso Civil Nicaraguence. [Nicaragua: Univerisidad Centroamericana].

García, A. W. (2017). Expediente Judicial digitalizado en el ambito del desarrollo de los procesos de alimentos. [Lima, Perú].

Gino, C. Y. (2012). El derecho a probar en la Tutela Ejecutiva. Derecho \& Sociedad, 147 153.

Guerra, C. M. (2018). Suma Procesal Civil. [Lima: Nomos \& Thesis E.I.R.L.].

Hernandez, S. R. (2014). Metodologìa de la Investigaciòn. Interamericana Editores S.A.

Hinostroza, M. A. (2016). Comentarios al Código Procesal Civil- Tomo II. [Lima: Instituto Pacífico]. 
Monroy, G. J. (2009). Teoria General del Proceso. [Lima: Comunitas].

Muñoz, C. Ó. (2014). La inmediación procesal. Análisis sobre su consagracion legal en el Código General del Proceso. Diálogos de Derecho y Política, 102-122.

Ochoa, B. M. (2005). La implementaciòn de la Oralidad en el Proceso Laboral debate en torno a la congetion Judicial. Dialogo de Saber, 69-94.

OVALLE, F. J. (2003). Los Alegatos como formalidad esencial de procedimiento. Cuestiones Cosntitucionales, 185-188.

Poémape, C. A. (2017). La Ejecución de las Sentencias en Procesos de Alimentos, en el Octavo Juzgado de Paz Letrado de Lima. [Lima, Perú].

Polanco, G. C. (2019). ¿Es posible la litigación oral en materia civil? Actualidad Civil, Al día con el derecho, 295-319.

Quezada, L. N. (2010). Metodología de la Investigación, estadistica aplicada a la investigación. [Lima: Macro EIRL].

Riega, V. Y. (2010). Investigaciòn y Desarrollo de Tesis en Derecho. [Lima: MAD Corp S.A.]. Rimachi, H. H. (2016). El deber del Juez de hacer cumplir sus decisiones: Una experiencia de buenas practicas en la ejecucion de los procesos de alimentos. Gaceta Constitucional y procesal Constitucional, 98, 137-153.

Robles Trejo, L., Robles Blacido, E., Sanchez Espinoza, R. \& Flores Leyva, V. (2015). Fundamentos de la Investigaciòn Jurìdica. [Lima: FFECAAT EIRL].

Romo, L. J. (2001). La ejecución de sentencias en el proceso civil como derecho a la tutela judicial efectivo. [Quito, Ecuador].

Santos. (2018). Calificacion de demandan y competencia en el proceso de alimentos. Gaceta Juridica.

Suntaxi, P. V. (2014). La Implementación de la Oralidad en el Procedimiento Civil. [Quito: Universidad Central del Ecuador].

Varsi, R. E. (2011). Tratado de Derecho de Familia. [Lima : Gaceta Jurídica].

Véscovi, E. (1999). Teoría General del Proceso. [Colombia: Temis S.A].

Zumaeta, M. P. (2015). Temas de Derecho Procesal Civil. [Lima: Jurista Editores].

\section{Conflicto de intereses}

El autor indica que no existen conflictos de interés. 
Vol. 1, Núm. 2, jul-dic, 2021

Copyright (c) 2021 Juan Fernando Deza Padilla

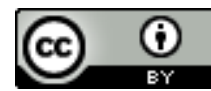

Este texto está protegido por una licencia CreativeCommons 4.0.

Usted es libre para Compartir — copiar y redistribuir el material en cualquier medio o formato- y Adaptar el documento - remezclar, transformar y crear a partir del material - para cualquier propósito, incluso para fines comerciales, siempre que cumpla la condición de:

Atribución: Usted debe dar crédito a la obra original de manera adecuada, proporcionar un enlace a la licencia, e indicar si se han realizado cambios. Puede hacerlo en cualquier forma razonable, pero no de forma tal que sugiera que tiene el apoyo del licenciante o lo recibe por el uso que hace de la obra.

Resumendelicencia - Textocompletodelalicencia

Citar como: Deza Padilla, J. F. (2021). Relación de oralidad y las decisiones judiciales en los procesos de alimentos tramitados en el segundo juzgado de paz letrado de Tarapoto, 2019. Revista Científica Ratio Iure, 1(2), 38-48. https://doi.org/10.51252/rcri.v1i2.196 